\title{
Ю.Н. Горго
}

Lиепропетровский государственный технический

универсттет железнодорожного транспорта

\section{Асимптотическое оптимальное управление элииптической системой с особыми характеристиками}

Стронтся и обосновывается решение двумерной задачи оптимального расиределенного управления эллиттичской системой с малым параметром при старшей производной в элемеитарной области - квадрате. Предполагается, что характеристики предельного урависиия совпадают с частьо границы области, а управлекие не ограничено.

Показано, то решения даниой задачи имеют нарастаюдие особенности на некоторьх внутренних множествах и, таким образом, задача является бисиигулярной.

1. Постановка задачи. Условия оптимальности.

Пусть в области $\Omega=\left\{\left(\mathrm{x}_{1}, \mathrm{x}_{2}\right): 0 \leq \mathrm{x}_{1} \leq 1,0 \leq \mathrm{x}_{2} \leq 1\right\}$ состоянио управляемой системы определяется как решение задачи Дирихле

$$
\begin{array}{r}
\varepsilon^{2} \Delta y\left(x_{1}, x_{2}\right)-a \frac{\partial}{\partial x_{2}} y\left(x_{1}, x_{2}\right)=u\left(x_{1}, x_{2}\right), \\
\left(x_{1} x_{2}\right) \in \Omega, y\left(x_{1} x_{2}\right)=0,\left(x_{1} x_{2}\right) \in \partial \Omega,
\end{array}
$$

где $\mathrm{u} \in \mathrm{L}_{2}(\Omega), \mathrm{y} \in \mathrm{H}_{0}^{1}(\Omega), 0 \leq \varepsilon \ll 1$, a $>0$.

Требуется найти такое управление $u$, чтобы

$$
I(u)=\inf _{u \in L_{2}(\Omega)} \int_{\Omega}\left[(y(u)-z(x))^{2}+v u^{2}\right] d x,
$$

rae $v=$ const $>0, z(x) \in L_{2}(\Omega)$.

Заметим, что задача (1)-(2). при фиксированном $\varepsilon$ имеет единственно решение [3].

Используя результаты [3], исходную задачу можно свести к задач математической физики вида

$$
\left\{\begin{array}{l}
\varepsilon^{2} \dot{\Delta y}-\quad a \frac{\partial}{\partial x_{2}} y=-v^{-1} p(x) \\
\varepsilon^{2} \Delta p+\quad a \frac{\partial}{\partial x_{2}} p=y(x)-z(x) \\
\left.y(x)\right|_{\partial \Omega}=\left.p(x)\right|_{\partial \Omega}=0
\end{array}\right.
$$

() Гopro К.H., 1998 
Гри этом оптимальное управление определяется формулой

$$
u=-v^{-1} p(x) \text {. }
$$

Z Асимптотика оптимального управления вне особьх многообразий.

E; дем считать, что в последующих рассуждениях имеет место

-ғедположение 1. Пусть $\mathrm{z}(\mathrm{x})$ - бесконечно дифференцируемая функция в 2. Будем искать регулярные составляюшие рядов в задаче (3) в виде

$$
\tilde{y}=\sum_{k=0}^{\infty} \varepsilon^{2 k-} y_{2 k}, \quad \bar{p}=\sum_{k=0}^{\infty} \varepsilon^{2 k} \cdot p_{2 k} .
$$

$\therefore 2$ ¿фициенты искомьх рядов легко определиктся из рекуррентнык - ж:тем, возникающих при подстановке рядов (4) в систему (3).

Для построения членов решения, удовлетворяющего граничным --овиям на верхней и нижней стороне области, необходимо привлекать -атранслойные разложения 'вдоль этих сторон квадрата. $\Omega$. Для этого इ三ョこем «быстрые» переменные $\tau_{0}=\varepsilon^{-2} \mathrm{x}_{2}, \tau_{1}=\varepsilon^{-2}\left(1-\mathrm{x}_{2}\right)$.

Тогда в окрестностях нижней и верхней границ строятся ряды

$$
\text { Sy }\left(\mathrm{x}_{1}, \tau_{0}\right), \operatorname{Sp}\left(\mathrm{x}_{1}, \tau_{0}\right) \text {, Sy }\left(\mathrm{x}_{1}, \tau_{1}\right), \operatorname{Sp}\left(\mathrm{x}_{1}, \tau_{1}\right) \text {. }
$$

Ә三ления систем,возникающих при подстановке рядов (5) в систему (3), в ¿̇стрьху координатах находим в классе погранфункций, стремящихся к $\because=0$ на бесконечности. Аккуратно выписывая внешние разложения и 泬山ни пограничного ряда, на основании их явного вида легко доказать, -2 имеет место

Лемма 1. Пусть выполнено предположение 1 , тогда ряды $\bar{y}+\mathrm{S}$ у и

=- $\mathrm{p}$ являются равномерным формальным асимптотическим разложением

三р.) решений системы (3) в области $\Omega$ и, кроме того, на верхней и - жней стороне квадрата $\Omega$ имеют место соотношения

$\therefore z_{2, x_{2}} \bar{y}+\left.A_{2 n, x_{1} \tau} S y\right|_{x_{2}=0}=O\left(e^{-\gamma_{1} / \varepsilon}\right),\left|A_{2 n_{, x_{1} x_{2}}} \bar{y}+A_{2 n_{2}, \tau} S y\right|_{x_{2}=1}=O\left(e^{-\gamma_{2} / \varepsilon}\right)$,

=- $A_{\text {: } x_{1} x_{2}}, R$ - частичная сумма ряда $R$ до $2 n$ слагаемого в переменньг

$\left.\because x_{2}\right)$, a $\gamma_{1}>0, \gamma_{2}>0$

$\equiv$ E.тренние разлокения.

- . :בируем невязки от внешнего разложения в окрестности вертикальньх -2. В ее окрестности введем переменную $\zeta=\mathrm{x}_{1} \varepsilon^{-1}$ и внутреннее ; зжение задачи (3) будем искать в виде

$$
\Gamma\left(\zeta, x_{2}\right)=\sum_{k=0}^{\infty} \varepsilon^{k} \Pi y_{k}\left(\zeta, x_{2}\right), \Pi p\left(\zeta, x_{2}\right)=\sum_{k=0}^{\infty} \varepsilon^{k} \Pi p_{k}\left(\zeta, x_{2}\right)
$$


Суммы рядов Пу и $\overline{\mathrm{y}}$, а также Пр.и $\overline{\mathrm{P}}$ должны удовлетворять нулевым граничным условиям на $\mathrm{x}_{2}=0$. и при $\mathrm{x}_{1}=\zeta=0$. Учитывая зто и представляя систему (3) в новых переменных, получаем системы вида $\begin{cases}\frac{\partial^{2} \Pi y_{0}\left(\zeta, x_{2}\right)}{\partial x_{0}^{2}}-a \cdot \frac{\partial}{\partial x_{2}} \Pi y_{0}\left(\zeta, x_{2}\right)=-v^{-1} \Pi p_{0}\left(\zeta, x_{2}\right), \\ \frac{\partial^{2} \Pi p_{0}\left(\zeta, x_{2}\right)}{\partial \partial^{2}}+a \frac{\partial}{\partial x_{2}} \Pi p_{0}\left(\zeta, x_{2}\right)=\Pi y_{0}\left(\zeta, x_{2}\right) ;\end{cases}$

$\left\{\begin{array}{l}\left.\frac{\partial^{2} \Pi y_{k}\left(\zeta, x_{2}\right)}{\partial \sigma^{2}}-a \frac{\partial}{\partial x_{2}} \Pi y_{k}\left(\zeta, x_{2}\right)=-1\right)^{-1} \Pi_{k}\left(\zeta, x_{2}\right)-\frac{\partial^{2} \Pi y_{k-2}\left(\zeta, x_{2}\right)}{\partial \gamma^{2}} \\ \frac{\partial^{2} \Pi p_{k}\left(\zeta, x_{2}\right)}{\partial \gamma_{0}^{2}}+a \frac{\partial}{\partial x_{2}} \Pi_{k}\left(\zeta, x_{2}\right)=I y_{0}\left(\zeta, x_{2}\right)-\frac{\partial^{2} \Pi p_{k-2}\left(\zeta, x_{2}\right)}{\partial \zeta^{2}}\end{array}\right.$

$\Pi \mathrm{yy}_{\mathbf{k}}(\zeta, 0)=\Pi \mathrm{p}_{\mathrm{k}}(\zeta, 1)=0$,

$\left\{\Pi_{2 \mathrm{k}}\left(0, \mathrm{x}_{2}\right)=-\mathrm{y}_{2 \mathrm{k}}\left(0, \mathrm{x}_{2}\right), \quad \Pi_{\mathrm{2k}+1}\left(0, \mathrm{x}_{2}\right)=0\right.$,

$\Pi p_{2 k}\left(0, x_{2}\right)=-p_{2 k}\left(0, x_{2}\right), \quad \Pi p_{2 k+1}\left(0, x_{2}\right)=0, k \geq 0$.

Здесь и в дальнейшем коэффициелы сы отрицательными индексами: считаем отрицательными.

Прежде чем приступать к последующим построениям проанализируем задачи (7)-(9).

Теорема 1. Функции $\Pi_{\mathrm{k}}$ и $\Pi_{\mathrm{k}}$ - решения задач (7)-(9)-при $\zeta \rightarrow \infty$ не превосходят по модулю величины $M_{k} \exp \left(-\alpha_{k} \zeta\right)$, где $M_{k}, \alpha_{k}>0$.

Доказательство данной теоремы основано на следующем. Используя преобразование Лапласа по переменной $\zeta$, системы (7)-(9) приводятся к системам обыкновенныг дифференциальных уравнений первого порядка относительно переменной $\mathrm{x}_{2}$. И собственно анализ поведения решения состоит в идее аналогичной [2]. А именно, пусть $\Pi y_{k}\left(\zeta, x_{2}\right)$ рассматриваемое решение, $\pi_{\mathrm{k}} \mathrm{y}$ - его изображение в новых переменных $\left(\zeta \rightarrow \rho, x_{2}\right)$, тогда при известных предположениях [4] имеет место равенство

$$
\lim _{\zeta \rightarrow \infty} \Gamma y_{k} \exp \left(-\alpha_{k} \zeta\right)=\lim _{\rho \rightarrow 0} \rho \pi_{k} y^{*} \frac{1}{\rho+\alpha_{k}} .
$$

Производя анализ свертки получаем утверждение теоремы.

Вообще говоря, на первый взгляд, задача нахождения решений Пу $\mathrm{g}_{\mathrm{k}}$ и $\Pi p_{\mathrm{k}}$ просто решается применением преобразования Лапласа. Тем не менее. оказывается, что она имеет характерные особенности в точке $(0,1)$. В самом 
z-2. так как из (3) $\frac{\partial \overline{\mathrm{p}}_{0}}{\partial \mathrm{x}_{2}}(0,1)=-\frac{\mathrm{y}_{0}(0,1)-\mathrm{z}(0,1)}{\mathrm{a}}$, то $\cdots$ в силу

$\left.\frac{\ddots=}{\ddots}\left(0, x_{2}\right)\right|_{x_{2}=1}=-\frac{y_{0}(0,1)-z(0,1)}{a}$. С другой стороны, из (8) имеем, что $\frac{\because \Pi p_{0}}{\delta_{2}}(\zeta, 1) \equiv 0$, тогда из (7) получаем, что $\left.\frac{\partial^{2} \Pi p_{0}}{\partial x_{2}^{2}}(\zeta, 1)\right|_{\zeta=0}=\frac{\bar{y}(0,1)}{a}$. :-зіми словами, функция Пр терпит разрыв производной в точке $(0,1)$. - жннна этой несогласованности в граничных условиях заключается в том, $\Longrightarrow$ мы ищем решения однородного уравнения, привлекая для граничньх \$ =авий решения соответствующего неоднородного уравнения. Очевидно, -0 если бы $z(0,1)=0$, то есть неоднородное уравнение в точке $(0,1)$ совпало $\varepsilon_{-i}$ с однородным, несогласованности в нулевом члене не было бы, однако с-а немедленно возникла в задачах для $\mathrm{k}>0$. Можно показать, что если бы -сшня $\mathrm{z}(\mathrm{x})$ вместе со всеми своими производными равнялась нулю в $-10(0,1)$, то задачи для $\Pi_{\mathrm{k}}, \mathrm{k} \geq 0$, в граничных условиях были бы c:-: гованы. Описанная особенность нулевого члена ряда для Пр $p_{k}$ растет с 三_.яением номера k: Так уже для определения коэффициента Пр 2 мы - қв:екаем в правой части операцию дифференцирования к разрывной с. вности, то в классе таких функций решение задач (7)-(9) не - - -венно. Для определения правильного решения необходимо с:-:三совать асимптотическое разложение $\Pi p_{k}$ с новым внутренним ба:лжением, определенным в точке $(0,1)$. С этой целью введем в :Еこизтривать внутрениее разложение вида

$$
\because(\xi, \eta)=\sum_{i=1}^{\infty} \varepsilon^{2 k} V y_{2 k}(\xi, \eta), V p(\xi, \eta)=\sum_{i=1}^{\infty} \varepsilon^{2 k} V p_{2 k}(\xi, \eta)
$$

$z=-110)$ начинаются с члена, содержащего $\varepsilon^{2}$, так как искомые решения у : - $三$ ны нулю з начале координат. Требование к рядам (10) следующее: - चлжжны в сумме с рядами (6) быть ф.а.р. однородной системы (3) z=-д точки $(0,1)$. Таким образом, ряды (10) должны быть решением

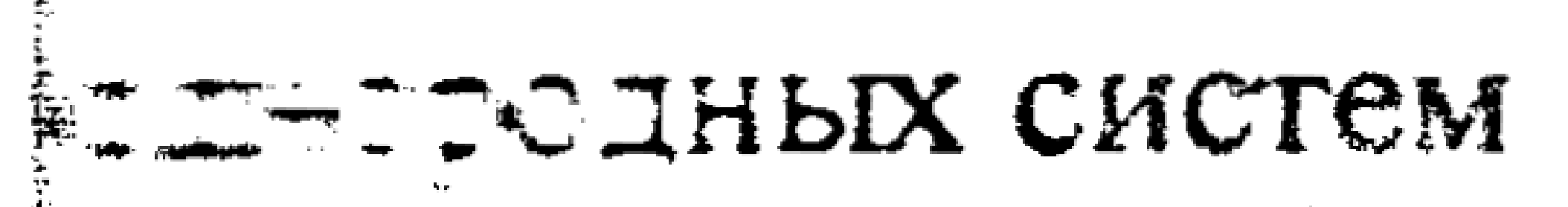

$$
\begin{aligned}
& \dot{\because}: \mathrm{k}+\mathrm{a} \frac{\partial}{\partial \eta} \mathrm{Vy}_{2 k}=-v^{-1} \mathrm{Vp}_{2(k-1)}, \\
& \dot{\because} F_{i k}-\frac{\partial}{\partial \eta} \mathrm{Vp}_{2 k}=\mathrm{Vy}_{2(\mathrm{k}-1)}
\end{aligned}
$$




$$
\begin{aligned}
& V y_{2 k}(\xi, 0)=0, \quad V p_{2 k}(\xi, 0)=0, \\
& V p_{2 k}(0, n)=-\sum_{j=1}^{k} \frac{\eta^{j}}{j !} \frac{\partial^{j p}{ }_{2(k-j)}(0,1)}{\partial \times i}, \\
& V_{y_{2 k}}(0, \eta)=-\sum_{j=1}^{k} \frac{\eta^{j}}{j !} \frac{\partial^{j y}{ }_{2(k-j)}(0,1)}{\partial x^{j}}
\end{aligned}
$$

Анализируя задачи (11)-(12), грежде всего заметим, что они не являют системами в полном смысле этого слова. Действительно, при каждо фиксированном k они распадаются на два, вообще говоря, независимь уравнения, решения которых легко выписываются посредством сходящих интегралов [1]. Таким образом, решение легко выписывается явньд образом в окрестности начала координат. Следующая наша задача построить общую часть асимптотик функций $\mathrm{Vp}_{\mathrm{k}}$ при $\xi^{2}+\eta^{2} \rightarrow \infty$ функций $\Pi_{p_{\mathrm{k}}}$ при $\zeta \rightarrow 0$. Для этого установим поведения функций $\mathrm{Vp}_{\mathrm{k}}$ бесконечности. Для упрощения дальнейших выкладок положим, что $\mathrm{a} \equiv$ Можно доказать [2], что имеет место

Теорема 2. Функции $V_{y_{k}}, V_{p_{k}}$. p шения задач (11)-(12) и все и проияодные по модулю не превосходят величины $M \exp (-\gamma \xi)$ пр $\xi \geq \mathrm{A} \eta+1$ для пюбого $\mathrm{A}>0$. Здесь $\gamma>0$ зависит от $\mathrm{A}$, а постоянная $\mathrm{M} \sigma$ номера $\mathrm{k}$ и порядка производной.

Из теоремы 2 можно заключить, что часть разложения V: конструктивно пригодная для процесса согласования на бесконечности может быть выделена только при условии $\eta \rightarrow \infty$. Однако получить эт. асимптотику из явного представления решения невозможно. Тем не мене используя математический аппарат [2], можно доказать, что для функци] $\mathrm{Vp}_{2}$ имеет место асимптотическое разложение $\mathrm{Vp}_{2}=\eta \sum_{\mathrm{j}=0}^{\infty} \eta^{-\mathrm{j} / 2} \Phi_{1, \mathrm{j}}(\Theta)$, $\mathrm{r}$ $\Theta=1 / 2 \frac{\xi}{\eta}, \quad$ функция $\Phi_{1, j}(\Theta) \in \mathrm{C}^{\infty}[0, \infty)$ и экспоненциально быстр стремится $\mathrm{x}$ нулю на бесконечности. Ряд $\mathrm{Vp}_{2}$ допускает почленно дифференцирование.

Наша следующая задача - найти асимттотический вид функци $\mathrm{Vy}_{2}$ при $\eta \rightarrow \infty$. Сама задача для $\mathrm{Vy}_{2}$ в силу знака "плюс" пере пройзводной имеет принципиально другую природу, однако этто неудобств удается обойти и доказать.

Теорема 3. Функции $\mathrm{Vy}_{\mathrm{k}} \mathrm{Vp}_{\mathrm{k}}$ - решения задач (11)-(12) при $\xi<\eta$ разлагаются в асимптотические ряды 


$$
V y_{2 k}=\eta^{K} \sum_{j=0}^{\infty} \eta^{-j / 2} \bar{\Phi}_{k, j}(\Theta), \quad V p_{2 k}=\eta^{k} \sum_{j=0}^{\infty} \eta^{-j / 2} \Phi_{k, j}(\Theta)
$$

Геперь мы уже можем приступить к построению ряда $\Pi_{\mathrm{k}}$.

Теорема 4: Существуют функция $\Pi_{\mathrm{k}}$ - решение систем (7)-(9), гторые имеют асимптотическое разложение вида

$$
\Pi p_{k}\left(\zeta, x_{2}\right)=x_{2}^{-k / 2} \sum_{j=1}^{\infty} x_{2}^{j} \Phi_{j, i}(\Theta), x_{2} \rightarrow 0 .
$$

Е-лы $П p_{\mathrm{k}}$ и $\mathrm{Vp}_{\mathrm{k}}$ согласованыв в смысле [4].

На основании теоремы 4 формируем составные разпожения функции $p$

$$
T_{N} p\left(x_{2}, x_{2}, \varepsilon\right)=A_{n, \zeta, x_{2}} \Pi p+A_{2 n, \xi, \eta} V p-A_{2 n, \xi, \eta} A_{n, \zeta, x_{2}} V p \text {. }
$$

В завершение этого этапа строим составнісе разложение $\mathrm{T}_{\mathrm{N}} \mathrm{y}$. Заметим - екде всего, что мы находим Vу как "вынужденное" решение, обладающее :-лтвами Vp, что является вполіне очевидным в силу того, что характер -: इедения Vу полностью определяется правой частью.

Будем строить составные асимттотические разложения (с.а.р.) для $5:-$ тии у в виде

$$
\mathrm{T}_{\mathrm{N}} \mathrm{y}\left(\mathrm{x}_{2}, \mathrm{x}_{2}, \varepsilon\right)=\mathrm{A}_{\mathrm{n}, \zeta, \mathrm{x}_{2}} \Pi y+\mathrm{A}_{2 n, \xi, \eta} \mathrm{Vy}-\mathrm{A}_{2 \mathrm{n}, \xi, \eta} \mathrm{A}_{n, \zeta, \mathrm{x}_{2}} \mathrm{Vy} .
$$

Следует отметить некоторую особенность выписанного с.а.p.

Замечание 1. Для функций Пу и Уу не выполняется условие :-:зсования вида [2]. Или, другими словами, не существует таких $-\because$ иножеств множества $\Omega$, чтобы поведение $\mathrm{T}_{\mathrm{N}}$ у на них описывалось 7.20 одним из слагаемых с.а.p.

В сушности это замечание имеет вполне естественное интуитивное - .;:ение. Действительно, мы находим функцию Vy как решение, вообще - इ. 2я, "искусственно" поставленной задачи, не связанной с функцией Пу

Iтя того чтобы построенные суммы были решениями однородной z--:ми (3) в области $\Omega$ и с их помощью можно ликвидировать невязку от z-三инх разложений на нижней, верхней и левой сторонах квалрата, эю- ходимо провести еце некоторые операции:

二 ф.а.p. $T_{K}$ у - компенсировать невязки около точки $(0,1)$;

: z . а.p. $T_{N}$ p - компенсировать невязки около точки $(0,0)$.

Гак как эти задачи аналогичны, то рассмотрим только точку $(0,0)$. -....и только обиую идею этого процесса. Невязку в функции р. на $\because \because: 3$

$$
Q y=\sum_{k=0}^{\infty} \varepsilon^{k} Q p_{k}(\zeta, \tau), \quad Q p=\sum_{k=0}^{\infty} \varepsilon^{k} Q p_{k}(\zeta, \tau)
$$


Для их членов получим системы обыкновенньх дифференциальньх уравнений, р решения которых удовлетворяют оценке $|\mathrm{Q}().(\zeta, \tau)|<\mathrm{M} \exp (-\delta(\zeta+\tau))$, так как подобным свойством обладают Пр .

X последнее, что осталось сделать, - это построить асимптотическое разложение Zp, которое удовлетворяло бы однородной скстеме (3) ликвидировало бы невязку от рядов $Q y, Q p$ при $\mathrm{x}_{1}=0$. Пусть

$$
\mathrm{Zy}(\xi, \eta)=\sum_{i=1}^{\infty} \varepsilon^{2 \mathrm{k}} \mathrm{Zy}_{2 \mathrm{k}}(\xi, \eta), \mathrm{Zp}(\xi, \eta)=\sum_{i=1}^{\infty} \varepsilon^{2 \mathrm{k}} \mathrm{Z} \mathrm{p}_{2 \mathrm{k}}(\xi, \eta)
$$

Как и для рядов Vy, Vр,задачи и краевые условия получаем в виде

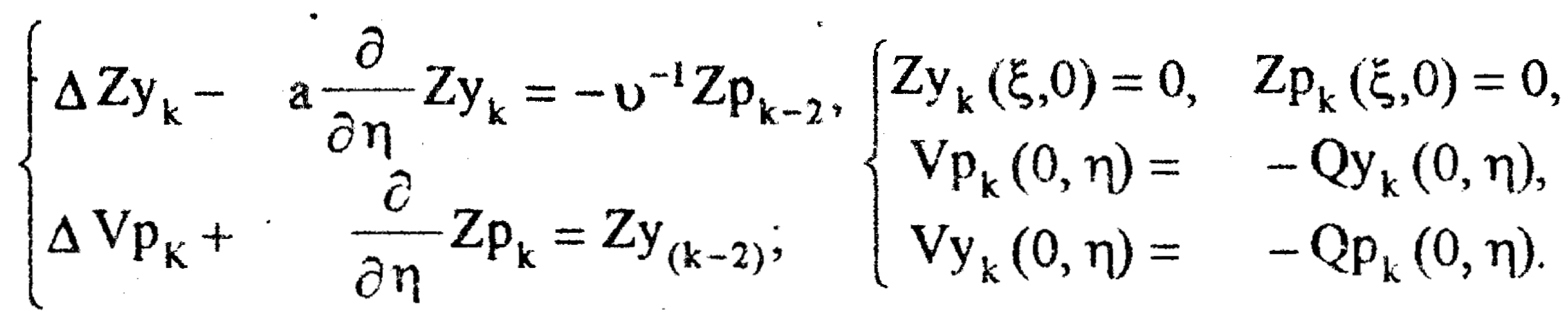

Решения этих задач можно записать в явном внде, привлекая известны формулы [5]. 'Отсюда немедленно вытекает, что функции $Z y, \quad Z$ экспоненциально быстро стремятся к нупю на бесконечности, так как этиу свойством обладают граничные условия исследуемых задач.

Считая, что построены ликвидационные ряды $\tilde{\mathrm{Q}}$ у $\tilde{\mathrm{Q}} \mathrm{p}, \tilde{\mathrm{Z}}$ у и $\tilde{\mathrm{Z}} \mathrm{p}$ для точки $(0,1)$, введем новые с.а.р

$$
Y_{N}=T y_{N}+Q y+\tilde{Q} y+\tilde{Z} y, \quad P_{N}=T p_{N}+Q p+\tilde{Q} p+\tilde{Z} p
$$

В завершение около границы $x_{2}=1$ строим ряды $\bar{Y}$ и и $\overline{\mathrm{P}}{ }_{\mathrm{N}}$, являющие решением однородной задачи (3), ликвидируюьщие невязки от внешне разложения на правой стороне квадрата и удовлетворяющие нулевы условиям на верхней и нижней сторонах. Возникаюшие особенност ликвидируем поочередно в точках $(1,0)$ и $(1,1)$ с помощью рядов $\mathrm{Vy}$. Vp и $\mathrm{Wy}^{*}$ и $\mathrm{Wp}^{*}$. Так как коэффициенты всех этих рядов быстро стремятся нулю при $\zeta \rightarrow \infty, \quad \xi \rightarrow \infty, \quad \zeta^{*}=\varepsilon^{-1}\left(1-x_{1}\right) \rightarrow \infty, \quad \xi^{*}=\varepsilon^{-2}\left(1-x_{1}\right) \rightarrow \infty$, ряды, построенные у границы $\mathrm{x}_{1}=0$, не оказывают влияния на гранично условие при $\mathrm{x}_{1}=1$ и обратно.

- Как итог приведенньх выше выкладок выпишем . полны асимттотические разложения во всей области $\Omega$ :

$y_{N}\left(x_{1}, x_{2}, \varepsilon\right)=\bar{y}+Y_{N}+\bar{Y}_{N}+S y, p_{N}\left(x_{1}, x_{2}, \varepsilon\right)=\bar{p}+P_{N}+\bar{p}_{N}+\dot{S}_{p}$ 
